Research Paper

\title{
FOXD3-induced miR-133a blocks progression and metastasis of colorectal cancer through regulating UBA2
}

\author{
Yuanfang Cheng1, Yajuan Wang1, Yuanzun Cheng'2, Quanzhong Yang1, Lejing Zhang1, Zhaoxi Li1, \\ Jiancheng Cheng ${ }^{3 凶}$ \\ 1. Sanquan College of Xinxiang Medical University, west of Changiiang Avenue, Pingyuan New Area, Xinxiang City, Henan Province, China. \\ 2. College of Nursing, LuoYang Polytechic, Luoyang, 471000 , Henan, China. \\ 3. Department of Cardiovascular Surgery, Zhengzhou Central Hospital Affiliated to Zhengzhou University, Zhengzhou 450000, China. \\ $\square$ Corresponding author: E-mail: 862512396@qq.com (JCC).
}

(c) The author(s). This is an open access article distributed under the terms of the Creative Commons Attribution License (https://creativecommons.org/licenses/by/4.0/). See http://ivyspring.com/terms for full terms and conditions.

Received: 2021.03.19; Accepted: 2021.07.28; Published: 2021.08.25

\begin{abstract}
Background and Aim: Some studies have verified that miR-133a played an inhibitory role in several cancers. Whereas, the effect of miRNA-133a in colorectal cancer (CRC) has not been fully elucidated. Our study aims to confirm UBA2 as a direct target gene of miRNA-133a and explore the upstream modulatory molecules of miR-133a. In addition, their impacts on the biological characteristics of CRC cells were assessed.

Methods: QRT-PCR analyzed miR-133a expression levels in colorectal cells including HCT116, SW48 cells and human normal colorectal cell line NCM460. A serial biological experiment assessed miR-133a effects on cell proliferation, migration, invasion and apoptosis capacities in HCT116 and SW48 cells. MiRNA targeting gene prediction and a dual luciferase assay were employed to confirm miR-133a-targeted UBA2. Transcription factors (TFs) FOXD3 was identified as an upstream regulator of miR-133a via JASPAR. The influence of miR-133a and FOXD3 on UBA2 expression was analyzed by qRT-PCR or western blot.

Results: miR-133a was lowly expressed in CRC cells. High miRNA-133a expression suppressed the proliferation, migration, invasion and enhanced apoptosis capacities of CRC cells. MiR-133a targeted the UBA2 mRNA 3'UTR area and reduced UBA2 protein expression. We also unveiled that FOXD3 high-expression significantly raised miR-133a expression and diminished UBA2 expression. We also discovered that high miR-133a expression augmented the effects of elevated FOXD3 expression on CRC cell proliferation, migration and invasion, whereas, low miR-133a expression generated the opposite outcomes.

Conclusion: FOXD3 induced miRNA-133a directly targeting UBA2 could affect the progression and growth of CRC.
\end{abstract}

Key words: MiR-133a; UBA2; Colorectal Cancer; FOXD3; GEO; ArrayExpress

\section{Introduction}

CRC is the third most commonly diagnosed carcinoma and cause of cancer-related death in the United States [1]. In spite of major advances in treatment containing surgery, chemotherapy and radiation treatment, the prognosis of CRC patients remains unfavorable [1]. It is urgently needed to explore the molecular mechanisms of CRC growth and progression, which may be underlying therapeutic targets for CRC therapy.

MiRNAs are small (20-22 nucleotides), single stranded, non-coding RNA molecules that play critical roles in various biological processes through post-transcriptionally regulating gene expression [2].
Evidence has demonstrated that miRNAs were tightly involved in the growth and development of tumors [3-5]. Huang et al. revealed a dissecting miRNA signature in colorectal cancer progression and metastasis [6]. Song et al. identified that FOXO3adriven miRNA signatures suppressed VEGF-A/NRP1 signaling and breast cancer metastasis [7]. Niu et al. revealed biological implications and clinical potential of metastasis-related miRNA in CRC [8]. Meanwhile, downregulation of miR-133a has been reported in several carcinomas, such as oral squamous cell carcinoma [9], gastric cancer [10], esophageal squamous cell carcinoma [11]. Besides, several reports 
discovered that TFs regulating miRNAs expression were strongly involved in carcinoma [12, 13]. Therefore, the deregulation of TFs-miRNAs may serve as a crucial factor of aberrant miRNA expression in tumor. We disclosed that miR-133a was obviously downregulated in CRC across bioinformatics methods. However, the therapeutic impact and mechanisms of TFs-miRNAs in the progression of CRC required to be further explored.

Herein we unveiled that miR-133a was significantly low-expressed in CRC tissue samples and cells. We determined UBA2 as a direct target of miR-133a, and the expression of UBA2 was decreased by miR-133a overexpression. Both knockdown of miR-133a and overexpression of UBA2 in CRC cell lines promoted cell proliferation, invasion and migration and inhibited apoptosis. In addition, to investigate the upstream regulatory association of miR-133a, TFs that modulated promoters of miR-133a was discovered through JASPAR. Taken together, our study indicated that miR-133a induced by TFs could regulate the growth and development of CRC by reducing UBA2.

\section{Methods and Materials}

\section{Bioinformatics data analyzing}

Firstly, The GEO and ArrayExpress databases were searched to obtain raw miRNAs and clinical data for each CRC sample. Afterward, the normalization of the raw miRNA data was executed across the Robust Multi-Array Average (RMA) and Linear Models for Microarray (LIMMA) algorithm [14]. Subsequently, Limma package [15] was adopted to unveil the differentially expressed miRNAs (DEMs) between the CRC tissues and normal tissues. $\mathrm{P}<0.05$ and $\log C>1.3$ exhibited a statistical significance. Then, we performed the overlapped analysis for CRC DEMs via E-MTAB-4036 in ArrayExpress database and GSE98406 in GEO database. Furthermore, the overlapped miRNAs were measured by adopting Venn diagram by vennDiagram package. Further, the volcano map was performed by utilizing ggplot2 package to test the DEMs' and differentially expressed genes' (DEGs) expression between the CRC tissues and the non-CRC tissues.

\section{Determination of miR-133a target genes}

The candidate target genes of miR-133a were determined via four bioinformatics software including miRWalk2.0 [16], TargetScan6.2 [17], miRanda [18] and RNA22 [19]. Furthermore, the predicted and validated target genes were adopted to intersect with the DEGs in GSE156355 and GSE50117 sets from GEO database. Likewise, the DEGs between the CRC tissues and the non-CRC controls were uncovered on the basis of the above screening strategy [14]. At last, the uncovered targeted genes were assessed across PCR and western blot assays.

\section{Cell Culture and Transfection}

Human normal colorectal cell line NCM460 and CRC cell lines HCT116, SW48 were purchased from the American Type Culture Collection. All cells were cultured in DMEM medium (Gibco, USA) supplemented with $10 \%$ FBS (Gibco, USA) at $37^{\circ} \mathrm{C}$ in a cell culture incubator containing $5 \% \mathrm{CO} 2$.

The miR-133a mimic, the mimics negative control (miR-NC) and miR-133a inhibitor were purchased from GenePharma (Shanghai, China). The pcDNA (Vector) and pcDNA-FOXD3 overexpression (FOXD3-OE) plasmids were purchased by GenePharma (Shanghai, China). The pcDNA (Vector) and pcDNA-UBA2 overexpression (UBA2-OE) plasmids were synthesized by GenePharma (Shanghai, China). All transfections were carried out by using Lipofectamine 3000 reagent (Invitrogen) following the manufacturer's protocol. Post transfection 48 hours, cells were collected for testing transfection efficiency via QRT-PCR analysis.

\section{Western Blot}

Total proteins were extracted via RIPA lysis buffer (Sangon Biotech) following the manufacturer's instruction. Then, the proteins were separated by applying 8-10\% SDS-PAGE gel (Beyotime, China). Following the separation process, proteins were transferred to PVDF membranes. After blocking with $5 \%$ nonfat milk for 1 hour, the membrane was incubated overnight at $4{ }^{\circ} \mathrm{C}$ with primary antibody. Signals were visualized applying chemiluminescence (Tanon, China). Primary antibodies employed for immunoblotting were exhibited as followings: (GAPDH, 1:5000, ab8245, Abcam; UBA2, 1:2000, ab185955, Abcam; FOXD3, 1:800, ab64807, Abcam).

\section{Quantitative reverse transcription-polymerase chain reaction (QRT-PCR)}

Total RNA extraction was conducted using TRIzol (Invitrogen, USA), according to the manufacturer's protocol. A reverse transcription kit (Invitrogen, USA) was used for reverse transcription in accordance with the manufacturer's instructions. Real-time PCR assay was carried out by employing the SYBR Premix Ex Taq II (TAKARA) on the basis of manufacturer's protocols. U6 and GAPDH were employed as the internal controls. Relative expression levels were measured applying the $2^{-\Delta \Delta \mathrm{Ct}}$ method. Primer sequences are placed in Table 1. 
Table 1. All special primers were used in this study

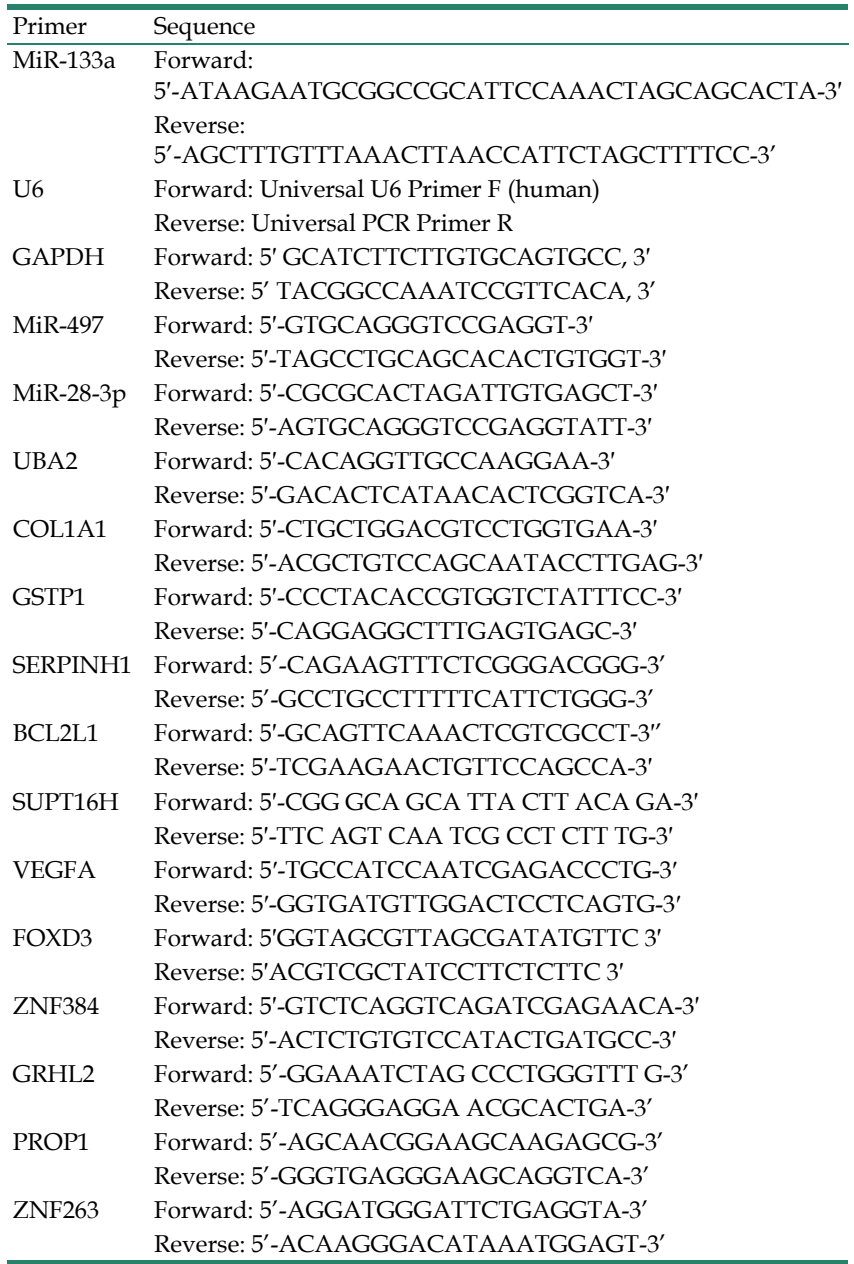

\section{Luciferase reporter assay}

Starbase databasewas was applied to predict the underlying targeting interplays between UBA2 and miR-133a. To identify the association between miR-133a and UBA2, luciferase reporter assay was carried out. HCT116, SW48 cells were transfected using PMIR-REPORT luciferase vector (GenePharma, Shanghai, China) with wild-type (WT)-UBA2-3'UTR or mutant (MUT)-UBA2-3'UTR, miR-133a mimic or miR-133a inhibitor (miR-133a-KD) or miR-NC. Post transfection for $48 \mathrm{~h}$, luciferase activities were measured across the Dual-Luciferase Reporter Assay System in accordance to manufacturer's instructions.

\section{Transwell assay}

Transwell assay were undertaken to test the migratory and invasive abilities of CRC cells. Cells were placed in the upper layer of the transwell chamber. Then, the lower chamber was filled with DMEM supplemented with 30\% FBS. Post culture for 24 hours, cells on the upper parts were wiped out, while the invaded cells in the lower compartments were fixed and stained using $0.1 \%$ crystal violet, and then counted according to a high-power microscope.

\section{Cell proliferation assay}

The proliferative capacity of CRC cells was assessed by 3-(4, 5-dimethylthiazol-2-yl)-2, 5diphenyltetrazolium bromide (MTT) assay in accordance to the manufacturer's instructions. Post transfection for $48 \mathrm{~h}$, the cells were added to 96-well plates and placed into an incubator at $37{ }^{\circ} \mathrm{C}$ with $5 \%$ $\mathrm{CO} 2$. At the indicated time point ( 24 hours, 48 hours, 72 hours, 96 hours and 120 hours), cells were harvested and interacted with 10 ul of MTT (5 $\mathrm{mg} / \mathrm{mL}$ ). Following 4 hours' incubation at $37^{\circ} \mathrm{C}, 100$ ul DMSO (per well) was used to dissolve the formosan. At last, a microplate reader was employed to detect the absorbance values at $490 \mathrm{~nm}$.

\section{Chromatin immunoprecipitation (ChIP) assay}

ChIP analysis of miR-133a was undertaken via a kit (Beyotime, Shanghai, China) in accordance to manufacturer's instructions. Briefly, FOXD3 antibody was used for immunoprecipitation of chromatin, and IgG was negative control and anti-RNA Polymerase II was utilized as positive control. Analysis of isolated DNA through RT-qPCR by applying gene-specific primers.

\section{Statistical analysis}

All statistical analyses were carried using GraphPad Prism 7.0. Data were exhibited as mean \pm SEM of at least 3 times of independent experiments and statistical significance of the differences was calculated with the one-way analysis of variance or Student t-test. $\mathrm{P}$ value $<0.05$ was considered to be statistically significant.

\section{Results}

MiR-133a expression was decreased and its high-expression was involved in favorable OS in CRC

We first performed overlapped miRNAs analysis between ArrayExpress database (E-MTAB-4036) and the GEO database (GSE98406) and found that seven miRNAs were DEMs in CRC tissues compared with non-CRC tissues Figure 1A. In addition, the clinical information of CRC patients in GEO and ArrayExpress was exhibited in Table S1. As a result of Figure 1B \& 1C, we exhibited the volcano plot of seven DEMs in GSE98406 and E-MTAB-4036 sets, respectively. Moreover, RT-qPCR assay showed that miR-133a level was lower in CRC cells (HCT116 and SW48) than that in NCM460 cells $(\mathrm{P}<0.05)$ (Figure 1D). Otherwise, we also analyzed the impact of miR-133a expression on CRC patients' prognosis in accordance to Kaplan-Meier analysis. downregulation 
of miR-133a was uncovered to be correlated with a shorter OS (Figure 1E) [20]. These obtained data suggested that miR-133a played a crucial role in the tumorigenesis of CRC.

\section{MiR-133a hinders proliferation, invasion, migration and facilitates the apoptosis of CRC cells}

MiR-133a was overexpressed or low-expressed in the two CRC cells to explore the influence of miR-133a on the CRC cell proliferation, invasion, migration and apoptosis. The results revealed that miR-133a level was elevated in CRC cells with miR-133a mimics, whereas miR-133a level was reduced in CRC cells with miR-133a inhibitor (Figure 2A), indicating that ectopic expression of miR-133a was successfully performed. Our data revealed that overexpression of miR-133a prominently blocked the proliferation, invasion as well as migration of the two CRC cells. Whereas, downregulation of miR-133a significantly promoted the proliferation, invasion and migration of the both CRC cells (Figure 2B-E), implying that miR-133a may serve as a cancer suppressor for CRC. On the other hand, miR-133a overexpression prominently blocked cell cycle progression and reinforced CRC cells apoptosis while miR-133a downregulation clearly promoted cell cycle progression and hindered CRC cells apoptosis (Figure 2F,G).
UBA2 is a direct target of miR-133a in CRC cells and restoration of UBA 2 rescues the cancer inhibitory role of miR-133a in CRC cells

Furthermore, we analyzed the latent mechanisms of miR-133a' inhibitory role in CRC. 13 common possible target genes were found out according to DEGs' overlapping analysis from GSE50117 and GSE156355 with predicted and validated target genes through miRWalk database (Figure 3A). As for the screened 13 common target genes, seven target genes expression in CRC tissues was revealed to be higher than that in control group (Figure 3B,C). In addition, in terms of the seven high expression target genes, UBA2 was the most prominently upregulated in the CRC cells in comparison to NCM460 cells (Figure 3D). To uncover the target of miR-133a in CRC cells, firstly we determined the possible target genes of miR-133a by applying miRWalk database for miRNA target prediction. As a result, UBA2 were selected for target analysis and the underlying binding site between miR-133a and UBA2 was predicted via miRWalk database (Figure 3F). To further explore whether UBA2 was a direct target of miR-133a, luciferase assay was conducted. These achieved data suggested that the luciferase activity of the UBA2-WT-3'-UTR plasmid in the CRC cell line was clearly decreased by miR-133a upregulation, and was obviously raised by miR-133a downregulation, however, the luciferase
A

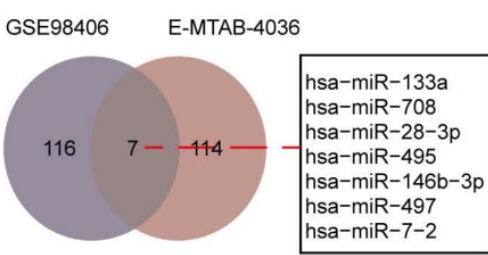

D

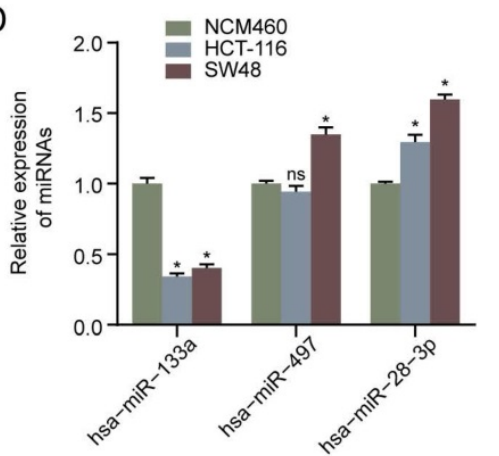

B

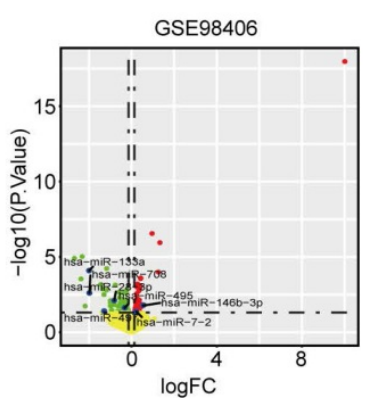

C

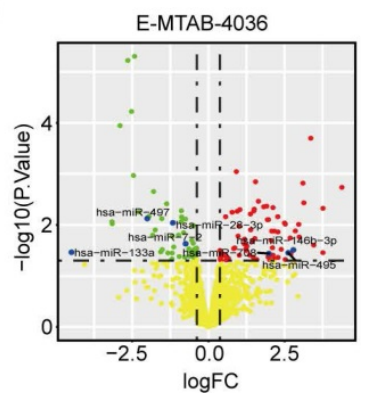

E

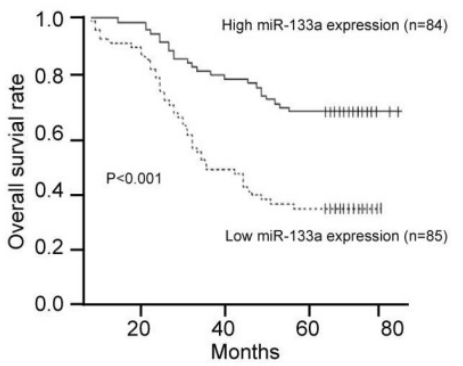

Figure 1. MiR-133a expression was diminished in CRC cell lines and tissues and miR-133a low-expression was associated poor prognosis. (A) Seven DEMs were selected in accordance to overlapping analysis of DEMs from GSE98406 and E-MTAB-4036 sets. (B,C) Volcano plot of Seven DEMs selected from GSE98406 and E-MTAB-4036 sets. (D) qRT-PCR assay of the three DEMs was performed in the two CRC cells and NCM460 cell. (E) Log-rank test for survival analysis according to miR-133a expression level. 
activity of UBA2-MUT-3'-UTR did not change (Figure $3 \mathrm{G})$, indicative of the direct correlation between miR-133a and the 3'UTR of UBA2 mRNA. In addition, the obtain data also indicated that decreased miR-133a expression augmented the proliferation, invasion and migration of the two CRC cells $(\mathrm{P}<0.05)$ (Figure 4A-E). The data also exhibited that miR-133a high-expression obviously attenuated cell cycle and enhanced CRC cells' apoptosis, which was prominently rescued by UBA2 high-expression (Figure 4F,G). Afterwards, we detected the relative expression levels of UBA2 with miR-133a high- expression or miR-133a low-expression in accordance to western blot analysis. These results exhibited that miR-133a upregulation evidently raised UBA2 expression and, while miR-133a low-expression had a reverse outcome (Figure 3E). Meanwhile, low UBA2 expression was correlated with good overall survival rate in CRC patients [21] (Figure S1A) and UBA2 was negatively associated with miR-133a albeit there is not significant in starbase database (Figure S1B). Based on these findings, we concluded that miR-133a might exert its role in CRC cells across directly regulating UBA2.
A

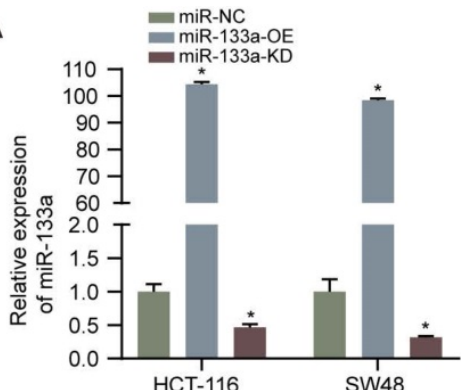

C

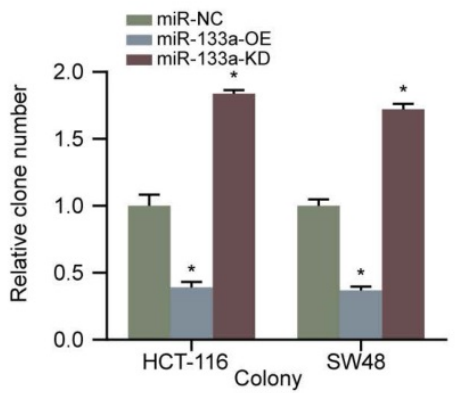

E
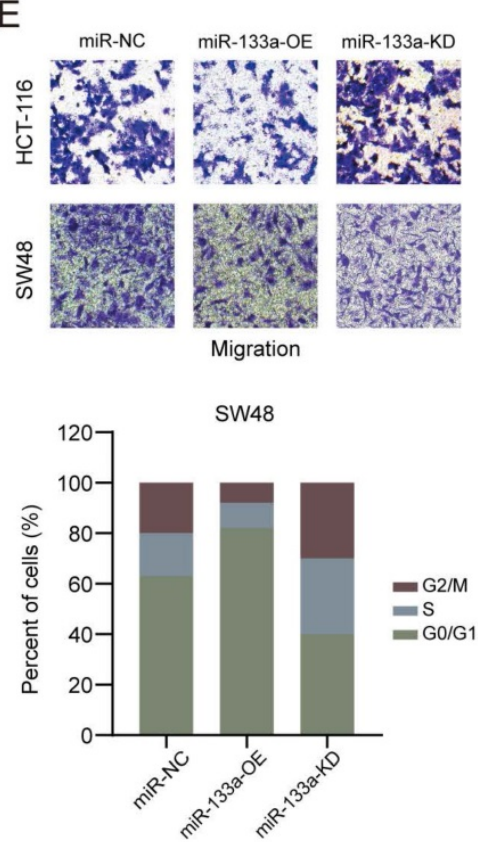

B

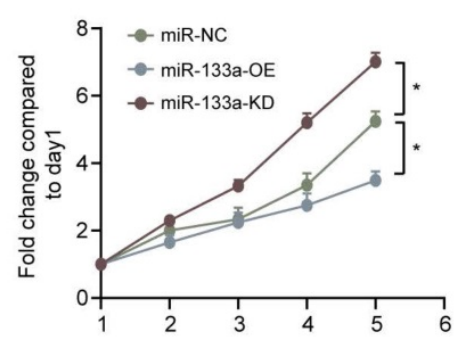

D

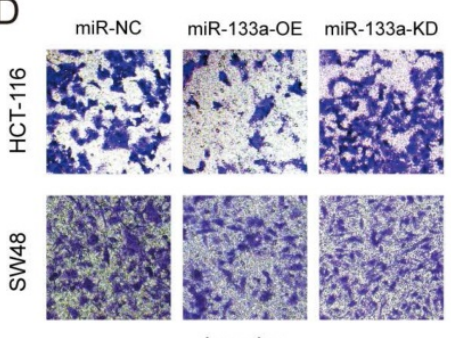

Invasion

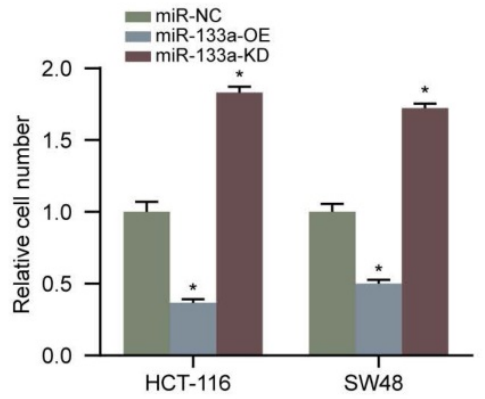

G
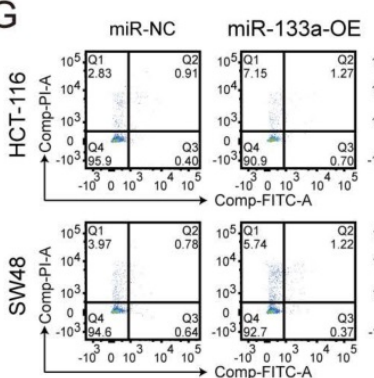

SW48
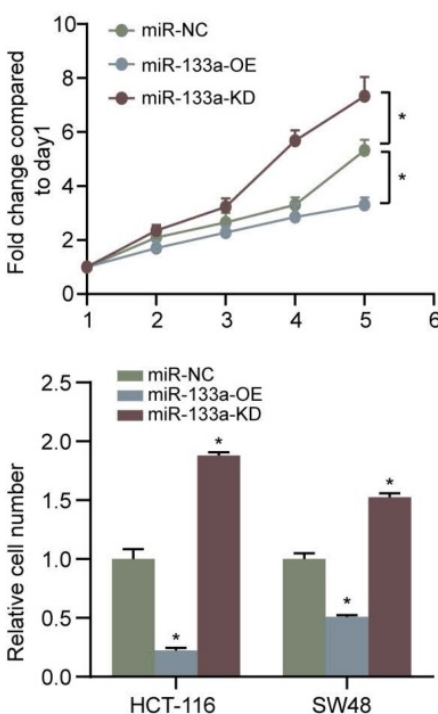

$\mathrm{F}$
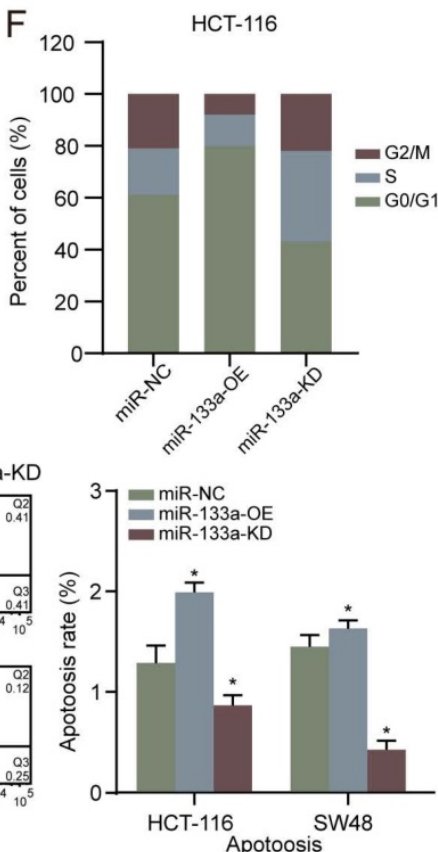

Figure 2. High miR-133a expression suppresses proliferation, invasion, migration, cell cycle and augments the apoptosis of CRC cells. (A) qRT-PCR assay for miR-133a expression level under miR-133a high-expression or reduced miR-133a expression in HCT116 and SW48 cells. (B, C) Cell proliferative capability was measured based on cell viability and colony formation with miR-133a high-expression or miR-133a low-expression in the both CRC cells. (D, E) Transwell assay was executed to analyze cell invasive and migratory ability with high miR-133a expression or diminished miR-133a expression in HCT116 and SW48 cells. (F, G) The impact of miR-133a low-expression or overexpression on cell cycle arrest and apoptosis in the two CRC cells was analyzed. 
A

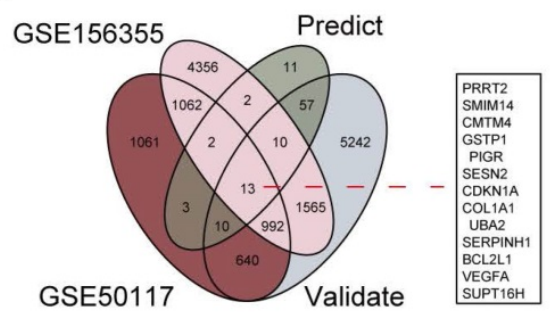

D

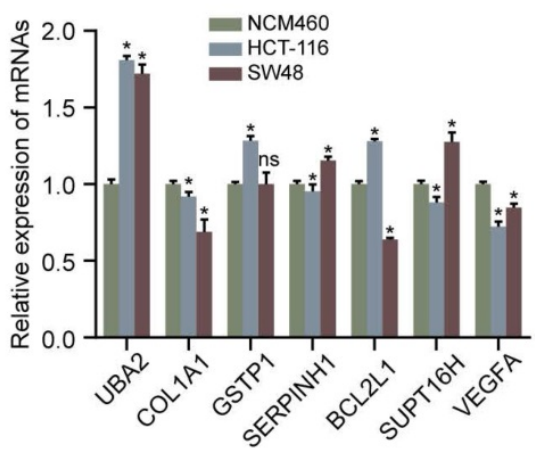

G

UBA2 3'UTR WT

5'....ACGACUAUACUUAUGGACCAAA ....3'

hsa-miR-133a

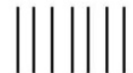

3'....GUCGACCAACUUCCCCUGGUUU....5

UBA2 3'UTR MUT

5'....ACGACUAUACUUAUCCUGGUUA....3'
B

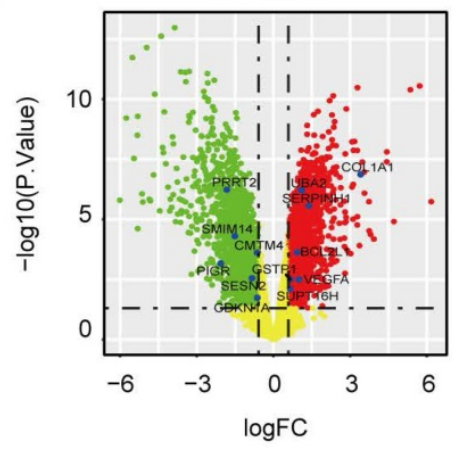

C

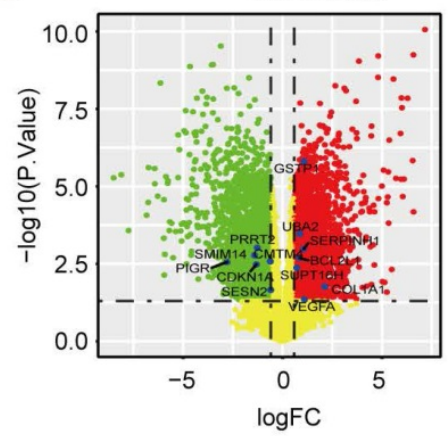

E

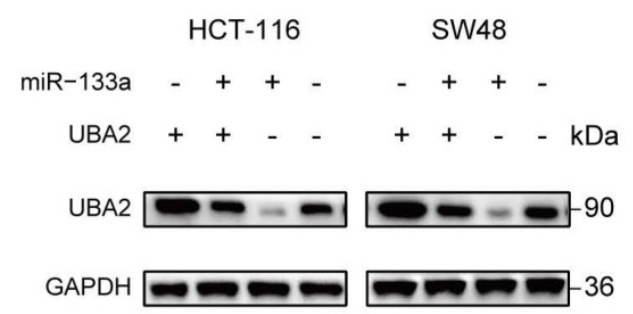

$\mathrm{H}$

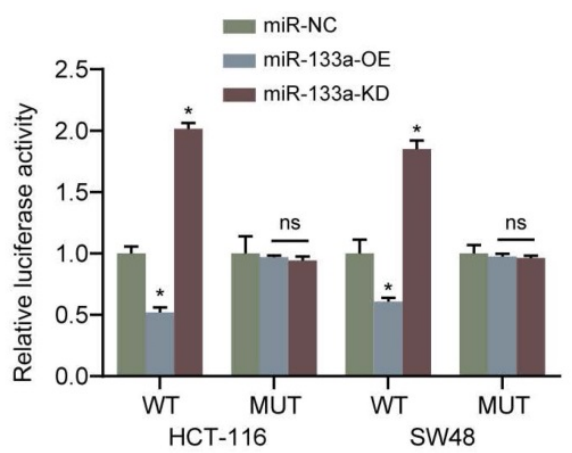

Figure 3. UBA2 is a direct target of miR-133a in CRC cells. (A) 13 common possible target genes were unveiled across DEGs' overlapping analysis from GSE50117 and GSE156355 with predicted and validated target genes. (B, C) Volcano plot of the unveiled 13 DEGs in GSE50117 and GSE156355, respectively. (D) QRT-PCR analysis for UBA2, COL1A1, GSTP1, SERPINH1, BCL2L1, SUPT16H and VEGFA in HCT116, SW48 and NCM460 cells. (E) Western blot assay for UBA2 with elevated miR-133a expression or reduced miR-133a expression. (F) MiR-133a binding site of UBA2's 3'-UTR. (G) PMIR-REPORT luciferase vector consisting of UBA2 3'UTR or a mutated type was co-transfected in the two CRC cells with miR-133a overexpression or miR-133a low-expression or miR-NC. Firefly luciferase activity was measured by applying Renilla luciferase activity.

\section{MiR-133a is a direct target of FOXD3}

To find out the upstream modulatory molecules of miR-133a, FOXD3 as a TF targeting promoters of miR-133a was identified by using JASPAR. Firstly, qRT-PCR assay was adopted to analyze the expression level of the top five potential TFs that could regulate the expression of miR-133a. We discovered that the expression level of FOXD3 was the most significantly high-expressed molecule in the two CRC cells compared with that in NCM460 cells (Figure 5A). Western blot analysis was adopted to measure the relative expression of FOXD3 and UBA2 with FOXD3 high-expression. The western blot analysis result manifested that UBA2 expression levels were decreased with FOXD3 upregulation in the two CRC cells (Figure 5B). As demonstrated in Figure 5C, QRT-PCR analysis proved that FOXD3 high-expression obviously increased the miR-133a expression in the two CRC cells. The evidence also revealed that high-expression of FOXD3 could suppress proliferation, invasion and migration of CRC cells, and this effect could be reversed by miR-133a downregulation $(\mathrm{P}<0.05)$ (Figure 5D-F).

Otherwise, ChIP assay was executed to verify whether FOXD3 transcriptionally regulated miR133a expression. Binding sites of FOXD3 inside the miR-133a promoter region was predicted according to JASPAR database. The ChIP assay identified the 
region at 414-425bp upstream of the pre-miR-133a promoter region as a target of FOXD3 (Figure 4F, G). Furthermore, luciferase activity in the WT miR-133a promoter was apparently increased under upregulation of FOXD3, and the effect was clearly rescued by miR-133a mimic. Whereas, no obvious difference was found under mutation of FOXD3 binding site at 414-425bp (Figure 5G). In addition, high FOXD3 expression was related with good overall survival rate (Figure S1C) and the expression of FOXD3 was positively correlatively with miR-133a (Figure S1D) in starbase database. Those results indicated that FOXD3-induced miR-133a block inhibits malignant progression of colorectal cancer through regulating UBA2 (Figure 6).
A

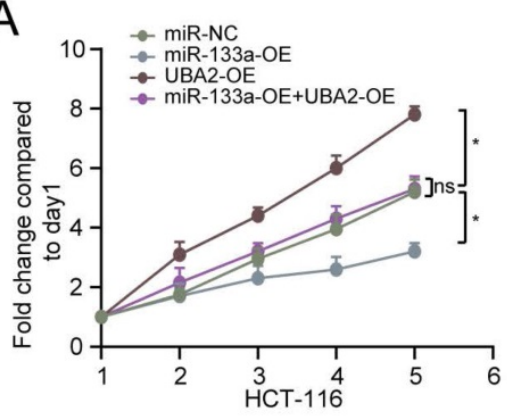

D
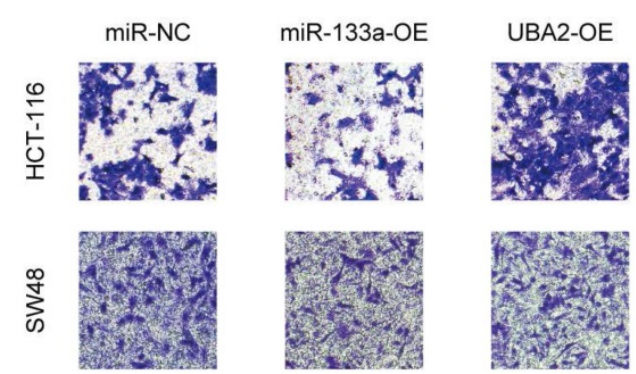

B
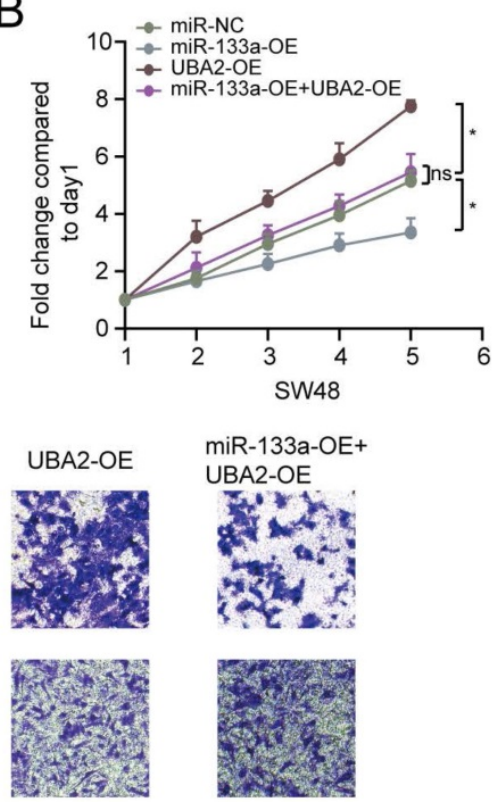

Invasion

E
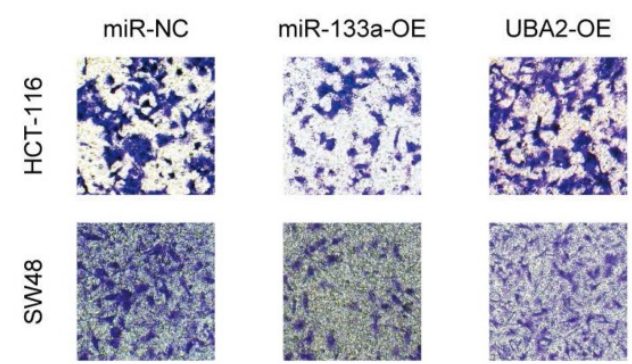

Migration

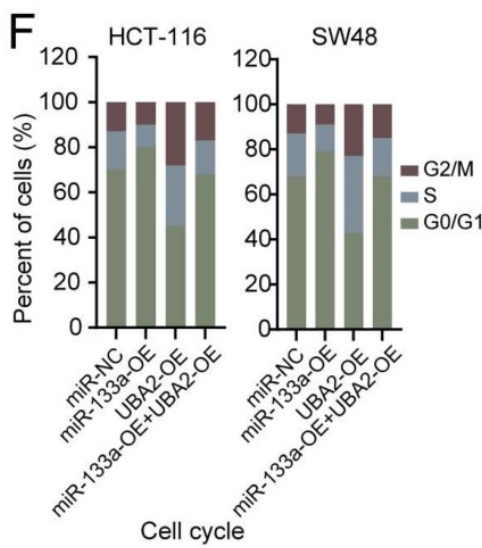

G
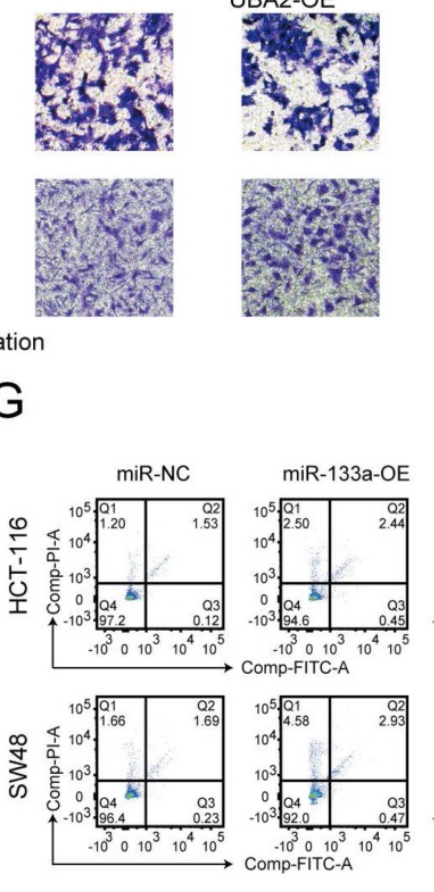

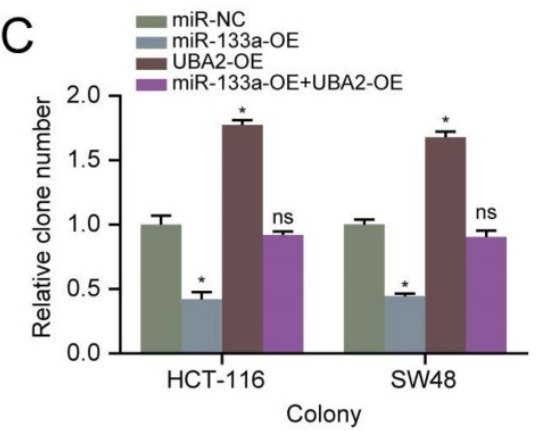

miR-133a-OE+ UBA2-OE
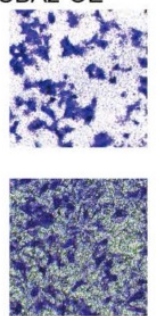

MiR-133a-OE+ UBA2-OE

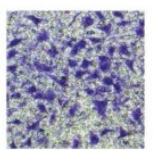

UBA2-OE
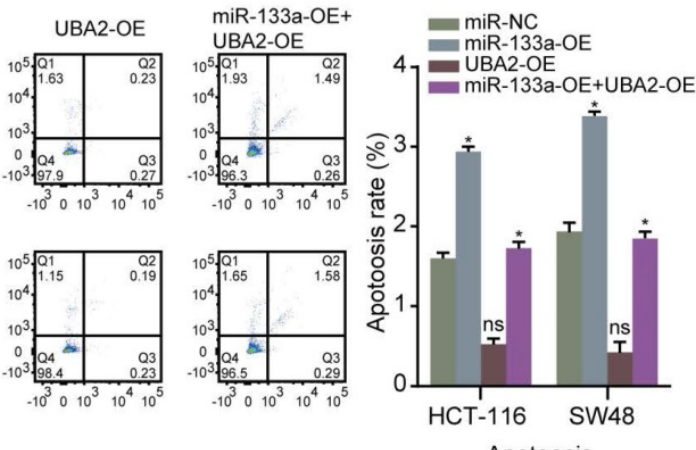

Figure 4. Restoration of UBA2 rescues the cancer inhibitory role of miR-133a in CRC cells. (A-C) The effect of miR-133a and UBA2 on CRC cell proliferative capability was analyzed according to cell viability and colony formation. (D, E) The effect of miR-133a and UBA2 on CRC cell invasion and migration capacity was assessed across transwell assay. (F, G) The effect of miR-133a and UBA2 on cycle arrest and apoptosis in CRC cell, respectively. 
A

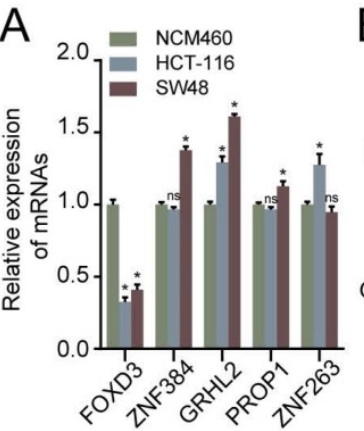

D
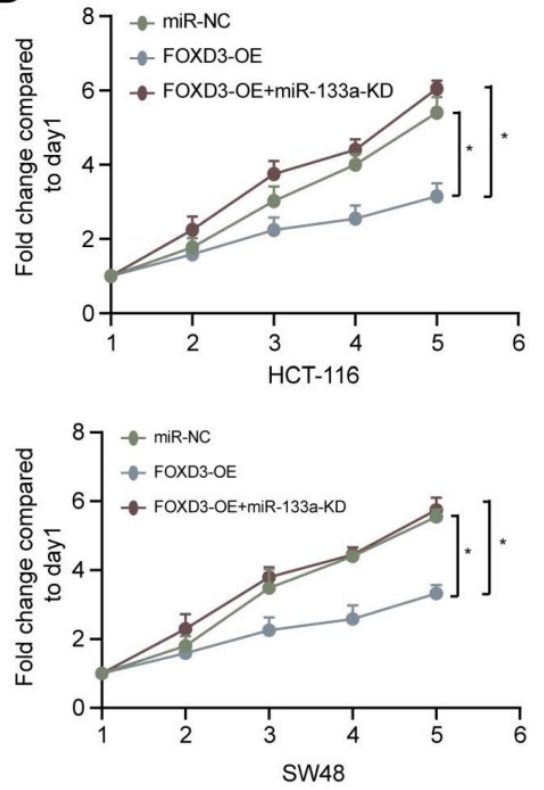

G

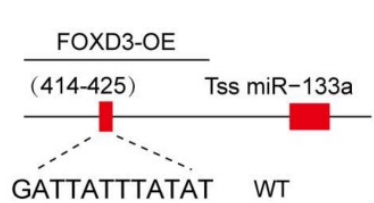

GUUUATUUATAT MUT
B
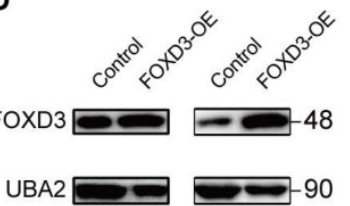

GAPDH -36

HCT-116 SW48 kDa

E

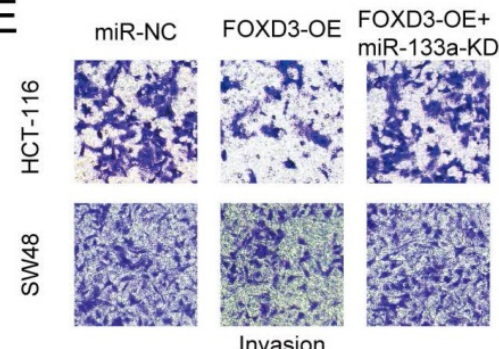

$\mathrm{F}$

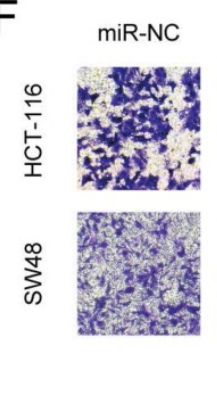

nvasion

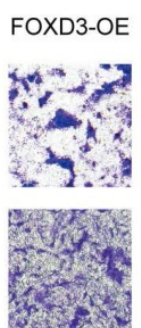

Migration
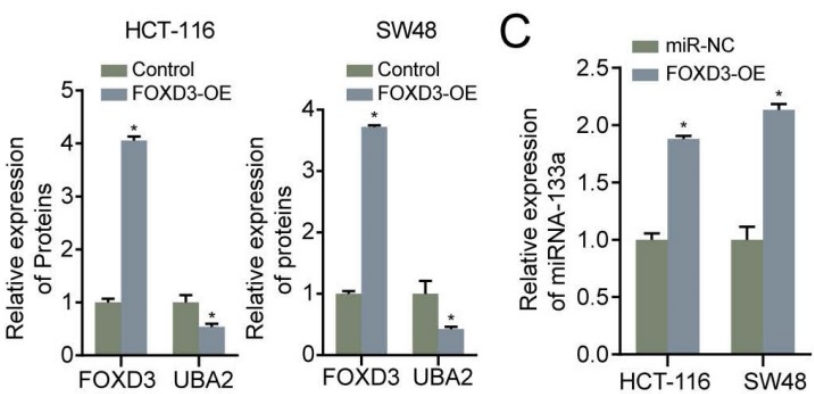
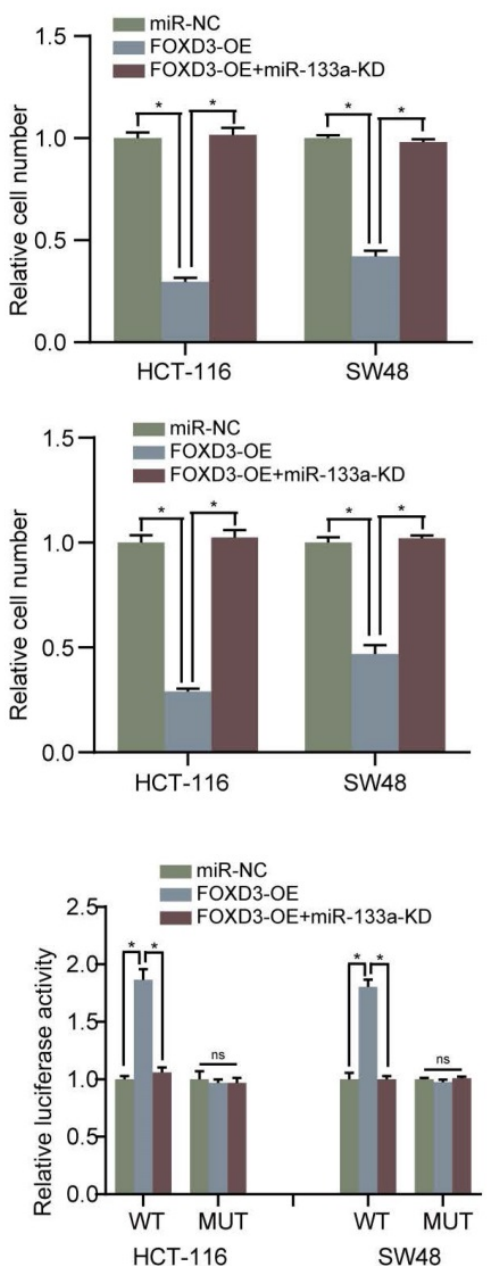

Figure 5. MiR-133a is a direct target of FOXD3. (A) qRT-PCR assay for FOXD3, ZNF384, GRHL2, PROP1 and ZNF263 expression level. (B) The effect of FOXD3 high-expression on miR-133a in the two CRC cells was measured via western blot assay. (C) MiR-133a was obviously raised under FOXD3 high-expression. (D) High FOXD3 expression significantly augmented the number of CRC-derived cell colonies formed, which was indicated by colony formation assay, and the effect was obviously reversed by miR-133a low-regulation. (E, F) Increased FOXD3 expression obviously heightened cell invasive and migratory capacity of HCT116 and SW48 cells, which was clearly rescued by diminished miR-133a expression based on transwell assay. (G) A schematic exhibited the proximal region of the miR-133a promoter and the region was detected by ChIP assay. (H) ChIP was adopted to validate the binding correlation between FOXD3 and the miR-133a promoter in the two CRC cells. (I) Luciferase activity was obviously augmented in the WT miR-133a promoter under high FOXD3 expression and the effect was obviously rescued by low miR-133a expression. Whereas, no obvious difference was found under mutation of FOXD3 binding site at $414-425$ bp. $* \mathrm{P}<0.05, * * \mathrm{P}<0.01$.

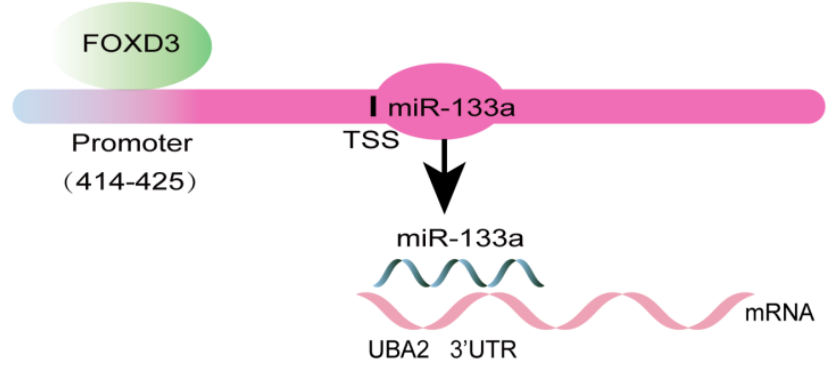

Figure 6. Schematic diagram of the present study. 


\section{Discussion}

Plenty of studies demonstrated that miRNAs were strongly correlated with tumorigenesis [22-24]. Otherwise, evidence suggested that miRNAs correlated with treatment of different types of tumors. For instance, Petrovic et al. revealed miRNAs as potential treatment targets and treatment options in cancer [25]. Jiang et al. found the clinical value of circular RNAs and autophagy-related miRNAs in the diagnosis and treatment of pancreatic cancer [26]. On the other hand, we found that miR-133a was remarkably reduced in CRC samples via a comprehensive bioinformatics approach, but the regulatory mechanism of the miR-133a in CRC keep still unclear.

In the present study, we investigate the function and mechanism of miR-133a in CRC. Consistent with our findings, miR-133a low-expression was also reported in gastric cancer [10], prostate cancer [27], esophageal cancer [28], and cervical cancer [29]. These results suggested that upregulation of miR-133a may serve as a repressor in various carcinomas. In the present paper, miR-133a high-expression was also verified to hinder the proliferation, invasion, migration and promote apoptosis of CRC cells (Figure 2). In this study, the data of Figure 1E exhibited that downregulation of miR-133a was tightly related to a shorter OS $(\mathrm{P}<0.05)$, suggesting that miR-133a may act as an effective biobiomarker for predicting CRC patients' prognosis. On the other hand, a lot of studies suggested that UBA2 functioned as an oncogenic gene. For example, Cheng et al. suggested that knockdown of UBA2 inhibited CRC cell invasion and migration by downregulation of the Wnt/betacatenin signaling pathway [30]. Li et al. indicated that UBA2 promoted cell migration and invasion through $W n t / \beta$-catenin signaling in gastric cancer [31], which were consistent with the findings in the present study. Additionally, the result of Figure 4 showed that UBA2 could promote the proliferation, invasion, migration and block apoptosis of CRC cells.

Numerous studies revealed that TFs were closely involved in different cancers. For example, Liu et al. found that TF c-Maf was a checkpoint that programs macrophages in lung cancer [32]. Jiang et al. indicated that TF NFAT5 promoted pancreatic cancer progression through transcription of PGK1 [33]. Seoane et al. suggested that POU1F1 TF promoted breast cancer metastasis by recruitment and polarization of macrophages [34]. Liu et al. revealed expression and clinical significance of TF 4 in epithelial ovarian cancer [35]. In this study, we first predicted TFs that targeted the promoter of miR-133a via JASPAR database. Following that, we analyzed the influence of high FOXD3 expression on miR-133a expression and miR-133a expression by qRT-PCR or western blot assay. Furthermore, the impact of increased FOXD3 expression on proliferation, invasion and migration ability of CRC cells was also analyzed. Based on the result of Figure 5D-F, high FOXD3 expression could suppress proliferation, invasion and migration of CRC cells.

\section{Conclusion}

In conclusion, these data manifested that FOXD3/miR-133a/UBA2 axis played a key role in the growth and progression of CRC. Our study suggested that FOXD3/miR-133a/UBA2 axis may have a great potential as a therapeutic target for CRC patients. The inhibitor of UBA2, and activator of FOXD3 and miR-133a may have a protective impact on CRC patients, which deserves further exploration and development.

\section{Supplementary Material}

Supplementary figure 1.

http://www.jcancer.org/v12p6145s1.pdf

Supplementary table 1 . The clinical information of

CRC patients in GEO and ArrayExpress.

http://www.jcancer.org/v12p6145s2.xlsx

\section{Acknowledgements}

This work was supported by research grants from 2018 Humanities and Social Sciences Research Project of the Education Department of Henan Province (NO. 2018-ZZJH-430).

\section{Data availability}

The data that support the findings of this study are available from the corresponding author upon reasonable request.

\section{Author contributions}

- Data curation: Yuanfang Cheng, Yajuan Wang;

- Formal analysis: Yuanfang Cheng, Jiancheng Cheng;

- Investigation: Yuanzun Cheng, Quanzhong Yang;

- Methodology: Lejing Zhang, Zhaoxi Li;

- Project administration: Jiancheng Cheng;

- Validation: Yuanfang Cheng;

- Writing - original draft: Yuanfang Cheng, Yajuan Wang, Jiancheng Cheng;

- Writing - review \& editing: Lejing Zhang, Yuanzun Cheng, Quanzhong Yang.

\section{Competing Interests}

The authors have declared that no competing 


\section{interest exists.}

\section{References}

1. Siegel RL, Miller KD, Jemal A. Cancer statistics, 2018. CA: a cancer journal for clinicians. 2018; 68: 7-30.

2. Fadaka AO, Pretorius A, Klein A. Biomarkers for Stratification in Colorectal Cancer: MicroRNAs. Cancer control : journal of the Moffitt Cancer Center. 2019; 26: 1073274819862784

3. Su WZ, Ren LF. MiRNA-199 inhibits malignant progression of lung cancer through mediating RGS17. European review for medical and pharmacological sciences. 2019; 23: 3390-400

4. Song J, Bai Z, Zhang Z. MicroRNAs are implicated in the initiation and progression of gastric cancer. Chinese medical journal. 2014; 127: 554-9.

5. Chen Z, Zhu J, Zhu Y, Wang J. MicroRNA-616 promotes the progression of ovarian cancer by targeting TIMP2. Oncology reports. 2018; 39: 2960-8.

6. Huang X, Zhu X, Yu Y, Zhu W, Jin L, Zhang X, et al. Dissecting miRNA signature in colorectal cancer progression and metastasis. Cancer letters. 2021; 501: 66-82.

7. Song Y, Zeng S, Zheng G, Chen D, Li P, Yang M, et al. FOXO3a-driven miRNA signatures suppresses VEGF-A/NRP1 signaling and breast cancer metastasis. Oncogene. 2021; 40: 777-90

8. Niu L, Yang W, Duan L, Wang X, Li Y, Xu C, et al. Biological Implications and Clinical Potential of Metastasis-Related miRNA in Colorectal Cancer. Molecular therapy Nucleic acids. 2021; 23: 42-54

9. He B, Lin X, Tian F, Yu W, Qiao B. MiR-133a-3p Inhibits Oral Squamous Cell Carcinoma (OSCC) Proliferation and Invasion by Suppressing COL1A1. Journal of cellular biochemistry. 2018; 119: 338-46.

10. Dong X, Su H, Jiang F, Li H, Shi G, Fan L. miR-133a, directly targeted USP39, suppresses cell proliferation and predicts prognosis of gastric cancer. Oncology letters. 2018; 15: 8311-8.

11. Yin Y, Du L, Li X, Zhang X, Gao Y. miR-133a-3p suppresses cell proliferation, migration, and invasion and promotes apoptosis in esophageal squamous cell carcinoma. Journal of cellular physiology. 2019; 234: 12757-70.

12. Li XL, Jones MF, Subramanian M, Lal A. Mutant p53 exerts oncogenic effects through microRNAs and their target gene networks. FEBS letters. 2014; 588: 2610-5.

13. Liao JM, Cao B, Zhou X, Lu H. New insights into p53 functions through its target microRNAs. Journal of molecular cell biology. 2014; 6: 206-13.

14. Diboun I, Wernisch L, Orengo CA, Koltzenburg M. Microarray analysis after RNA amplification can detect pronounced differences in gene expression using limma. BMC genomics. 2006; 7: 252.

15. Song S, Ajani JA. The role of microRNAs in cancers of the upper gastrointestinal tract. Nature reviews Gastroenterology \& hepatology. 2013; 10: 109-18.

16. Dweep H, Gretz N, Sticht C. miRWalk database for miRNA-target interactions. Methods in molecular biology (Clifton, NJ). 2014; 1182: 289-305.

17. Friedman RC, Farh KK, Burge CB, Bartel DP. Most mammalian mRNAs are conserved targets of microRNAs. Genome research. 2009; 19: 92-105.

18. Betel D, Koppal A, Agius P, Sander C, Leslie C. Comprehensive modeling of microRNA targets predicts functional non-conserved and non-canonical sites. Genome biology. 2010; 11: R90.

19. Kertesz M, Iovino N, Unnerstall U, Gaul U, Segal E. The role of site accessibility in microRNA target recognition. Nature genetics. 2007; 39: 1278-84.

20. Wang LL, Du LT, Li J, Liu YM, Qu AL, Yang YM, et al. Decreased expression of miR-133a correlates with poor prognosis in colorectal cancer patients. World journal of gastroenterology. 2014; 20: 11340-6.

21. He P, Sun $X$, Cheng HJ, Zou YB, Wang Q, Zhou CL, et al. UBA2 promotes proliferation of colorectal cancer. Molecular medicine reports. 2018; 18: 5552-62.

22. Jiang L, Hermeking H. miR-34a and miR-34b/c Suppress Intestinal Tumorigenesis. Cancer research. 2017; 77: 2746-58.

23. Lv C, Li F, Li X, Tian Y, Zhang Y, Sheng X, et al. MiR-31 promotes mammary stem cell expansion and breast tumorigenesis by suppressing Wnt signaling antagonists. Nature communications. 2017; 8: 1036

24. Fuziwara CS, Kimura ET. MicroRNAs in thyroid development, function and tumorigenesis. Molecular and cellular endocrinology. 2017; 456: 44-50.

25. Petrovic N, Ergun S. miRNAs as Potential Treatment Targets and Treatment Options in Cancer. Molecular diagnosis \& therapy. 2018; 22: 157-68.

26. Jiang PC, Bu SR. Clinical value of circular RNAs and autophagy-related miRNAs in the diagnosis and treatment of pancreatic cancer. Hepatobiliary \& pancreatic diseases international : HBPD INT. 2019; 18: 511-6.

27. Tang Y, Pan J, Huang S, Peng X, Zou X, Luo Y, et al. Downregulation of miR-133a-3p promotes prostate cancer bone metastasis via activating PI3K/AKT signaling. Journal of experimental \& clinical cancer research : CR. 2018; 37: 160 .

28. Li S, Qin X, Li Y, Zhang X, Niu R, Zhang H, et al. MiR-133a suppresses the migration and invasion of esophageal cancer cells by targeting the EMT regulator SOX4. American journal of translational research. 2015; 7: 1390-403.

29. Song X, Shi B, Huang K, Zhang W. miR-133a inhibits cervical cancer growth by targeting EGFR. Oncology reports. 2015; 34: 1573-80.

30. Cheng H, Sun X, Li J, He P, Liu W, Meng X. Knockdown of Uba2 inhibits colorectal cancer cell invasion and migration through downregulation of the
Wnt/ $\beta$-catenin signaling pathway. Journal of cellular biochemistry. 2018; 119: 6914-25.

31. Li J, Sun $X$, He P, Liu WQ, Zou YB, Wang Q, et al. Ubiquitin-like modifier activating enzyme 2 promotes cell migration and invasion through Wnt/ $\beta$-catenin signaling in gastric cancer. World journal of gastroenterology. 2018; 24: 4773-86.

32. Liu M, Tong Z, Ding C, Luo F, Wu S, Wu C, et al. Transcription factor c-Maf is a checkpoint that programs macrophages in lung cancer. The Journal of clinical investigation. 2020; 130: 2081-96.

33. Jiang Y, He R, Jiang Y, Liu D, Tao L, Yang M, et al. Transcription factor NFAT5 contributes to the glycolytic phenotype rewiring and pancreatic cancer progression via transcription of PGK1. Cell death \& disease. 2019; 10: 948.

34. Seoane S, Martinez-Ordoñez A, Eiro N, Cabezas-Sainz P, Garcia-Caballero L, Gonzalez LO, et al. POU1F1 transcription factor promotes breast cancer metastasis via recruitment and polarization of macrophages. The Journal of pathology. 2019; 249: 381-94.

35. Liu L, Zeng Z, Yi J, Zuo L, Lv J, Yuan J, et al. Expression and clinical significance of transcription factor 4 (TCF4) in epithelial ovarian cancer. Cancer biomarkers: section A of Disease markers. 2019; 24: 213-21. 